\title{
Investigation of irregularity of flow distribution across the tube bundle in shell-and-tube heat exchanger with segmental baffles using PIV method
}

\author{
Grzegorz Ligus ${ }^{1, *}$, and Szymon Kołodziej ${ }^{2}$ \\ ${ }^{1}$ Opole University of Technology, Department of Environmental Engineering, 5 Mikolajczyka St., Opole, Poland \\ ${ }^{2}$ Opole University of Technology, Department of Vehicles, 5 Mikolajczyka St., Opole, Poland
}

\begin{abstract}
This paper presents the results of an experimental study into flow through a tube bundle applied in shell-and-tube heat exchanger with segmental baffles built on an industrial scale. The reported research applied Digital Particle Image Velocimetry (PIV) technique. The study offered the determination of the velocity vector fields, which were subsequently used for identification and measurement of irregularity of flow distribution across the tube bundle. Differences in the intensity of local flow velocities in shell side were identified by velocity profiles analysis. Furthermore correlation between the map of local velocity and its location in particular tube rows was described. As a result, the three main regions of flow velocity variation throughout cross-section of the shell side were identified.
\end{abstract}

\section{Introduction}

Among the equipment applied for heat exchange, shelland-tube heat exchangers hold an established position in the industry. They are successfully applied in the processes involving heating, cooling, heat recovery, boiling, distillation and condensation. The wide range of the application of this type of exchangers comes as a consequence of several important advantages of such equipment: high value of the heat transfer coefficient in relation to the space that is occupied by them accompanied by their low weight, as well as established manufacturing and exploitation technology combined with considerable flexibility in terms of the operating parameters of this type of apparatus [1]. The shell side region is occupied by various type of baffles, which are applied with the objective of increasing the efficiency of the heat transfer in the shell-and-tube heat exchangers. Such baffles are based on a number of design improvements, which play the role of increasing the turbulence of the medium that flows around the tube bundle. As a consequence, heat exchange coefficient increases as well [2,3]. The basic types of baffles including segmental, grid, disk, helicoidal and others differ in terms of the hydrodynamic characteristics, practical application and the cost of production. Among these types of baffles, the considerable applicability coupled with the high increase of the heat exchange coefficient can be achieved due to the application of segmental baffles [1]. The only problems associated with their application are associated with the considerable pressure drop resulting from the disturbance generated by the flow through the shell side region [4,5]. This directly affects the increase of the exploitation cost of the heat exchangers. In addition, the existence of multiple rows of tubes and baffles in the shell side leads to a further additional interference in the velocity of the liquid in the shell side [6,7]. The differences in the intensity of local flow velocities consequently leads to the occurrence of various heat transfer coefficients for particular tube rows. In the consideration of the above, it is important to relate the map of local velocity in the shell side to the particular tube rows. The use of the Digital Particle Image Velocimetry (PIV) method forms one of the measurement methods with a potential application in the identification and measurement of the flow velocity in the shell side. PIV applies non-invasive, optical measurement techniques useful in the experimental research of aero- and hydrodynamic parameters [8]. An adequately implemented measurement conducted by use of this method is considered to be able to provide highly measurable results, even in applications with a complex geometry $[9,10,11,12]$. This statement definitely finds an application in the case of shell side of a heat exchanger discussed in this paper.

\section{Measurement setup and methodology}

The use of Digital Particle Image Velocimetry (PIV) involved the necessity to develop and build a heat exchanger with a maximum possible transparency of the shell and tube bundle $[8,13]$. This task was achieved by preparing a model of a heat exchanger produced from polymethyl methacrylate (PMMA). The tested model of the heat exchanger was built from a shell with an internal diameter of $240 \mathrm{~mm}$ and a length of $2000 \mathrm{~mm}$ and

\footnotetext{
*Corresponding author: g.ligus@po.opole.pl
} 
comprised a tube bundle with the internal diameter of 20 $\mathrm{mm}$. The testing applied a rectangular tube layout with the pitch ratio of 0.625 . Ten segmental baffles were installed along the tube bundle, whose baffle cut was equal to $25 \%$. To eliminate the gas phase from the system, the top section of the uppermost baffle comprised an air vent. The above model of a heat exchanger forms the principal part of the experimental set-up (Fig. 1). The arrangement of the tube bundle along with the markings used in the investigation is shown in Fig. 2.

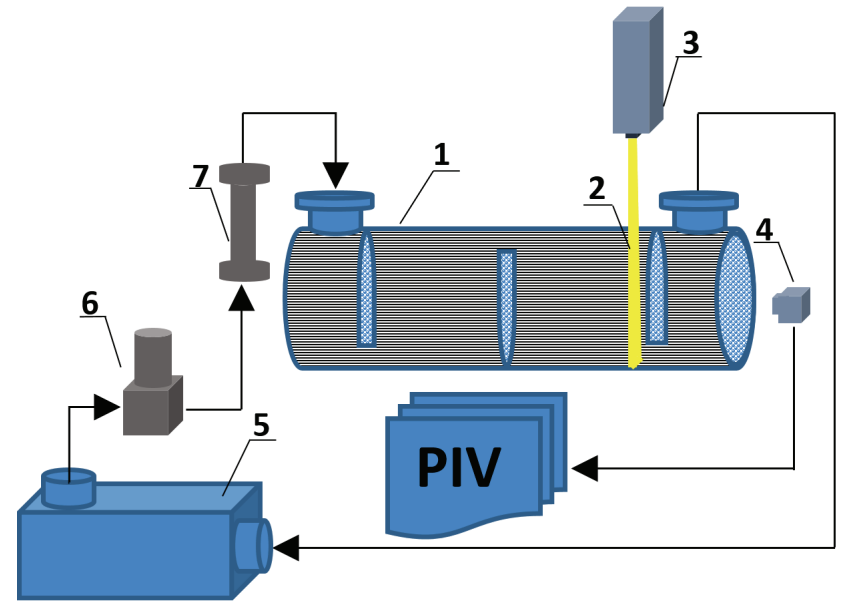

Fig. 1. Experimental set-up with cross section of the heat exchanger: 1. Model of heat exchanger, 2. Laser sheet, 3. Laser, 4. Camera CCD, 5. Water tank, 6. Pump, 7. Flowmeter.

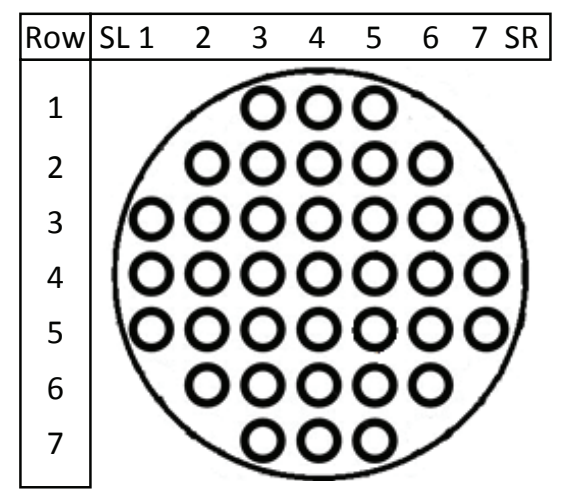

Fig. 2. Arrangement of the tube bundle: 1, 2, 3, 4, 5, 6, 7 numbers of the tubes in a row; SL - left side of the shell, SR right side of the shell.

Water stored in a tank with the capacity of $200 \mathrm{dm}^{3}$ played the role of the circulating medium. The circulation of the medium through the heat exchanger was possible due to the use of a multistage water pump, which was controlled by a signal transmitted from the electromagnetic flowmeter. The applied method also imposed the need to use seeding particles in the circulating medium. The presented study applied $3 \mathrm{~g}$ PSP polyamide with the particle diameter of $20 \mu \mathrm{m}$ and a density of $1.03 \mathrm{~g} / \mathrm{cm}^{3}$. These particles are characterized by a considerable value of the refractive index, as it is equal to 1.5. Due to the above characteristics, in many works $[13,14,15]$, PSP particles are recommended for applications in systems including water phase flow in particular for the case of the complex stream trajectory. In order to obtain a constant water homogenization with seeding particles, an agitator was installed in the tank. The studies into hydrodynamic parameters of the heat exchanger model were conducted for three flow rates of the medium equal to: $5.0 \mathrm{~m}^{3} / \mathrm{h}, 7.5 \mathrm{~m}^{3} / \mathrm{h}$ and $10 \mathrm{~m}^{3} / \mathrm{h}$. A CCD EO-4M Dantec Dynamics FlowSence camera was located in the optical track of the set-up, which provided the registration of the shell side region of the heat exchanger in the transverse direction across the tube bundle before the final baffle. The imaging space occupied the entire cross-sectional field of the heat exchanger. The registration was performed at a resolution of $2048 \times 2048$ pixels in the double frame mode with a frequency of $10 \mathrm{~Hz}$. The selection of the time between pulses was made on the basis of initial tests and it was set at the level of $500 \mu \mathrm{s}$ for all flow conditions. Each of the measurement series comprised 250 double frame images. The area that was subjected for observation purposes was illuminated by a laser light (Nd:YAG Dantec Dynamics DualPower TR) by application of the laser sheet technique. The laser generated a coherent light beam with a length of $532 \mathrm{~nm}$ and pulse energy of $1200 \mathrm{~mJ}$. During the research, the light sheet crossed the shell tube at distance of $10 \mathrm{~mm}$ before the final baffle. The calculations with regard to the velocity vector fields performed by application of the PIV method were conducted in the rectangular regions of interest, each of which involved the space between the adjacent tube rows that was included to the greatest possible extent. The velocity profiles were determined in the symmetry axes of the measurement regions of interest. The analysis applying PIV was conducted for each of the 250 pairs of the registered double frame images. Fig. 3 contains an overview of the PIV methodology applied during the calculations and analysis. These calculations were performed by application of the Dantec Dynamics Dynamic Studio 4.10. The determination of the velocity vector fields applied Adaptive Correlation algorithm, and it involved the measurement of the displacement between two patterns of pixel sets in selected image section, which were found to be representative for the flow images. In turn, they were represented by two-dimensional greylevel distributions. On the basis of the correlations of two subsequent flow images with the specific pixel patterns and by application of Fast Fourier Transforms, displacement vectors of images with a given grey-level distribution were calculated. This data resulted in development of a vector velocity field. The Fast Fourier Transform forms a calculation algorithm of the Discreet Fourier Transform (DFT) expressed by the formula:

$$
F(i, k)=\beta_{O} \sum_{m=0}^{M-1} \sum_{n=0}^{N-1} O(m, n) \exp \left(\frac{-j 2 \pi m i}{M}\right) \exp \left(\frac{-j 2 \pi n k}{N}\right)
$$

for $\mathrm{m}=0,1, \ldots, \mathrm{M}-1 ; \mathrm{n}=0,1, \ldots, \mathrm{N}-1$

where:

$$
\mathrm{O}(\mathrm{m}, \mathrm{n})=\beta_{\mathrm{F}} \sum_{\mathrm{i}=0}^{\mathrm{M}-1} \sum_{\mathrm{k}=0}^{\mathrm{N}-1} \mathrm{~F}(\mathrm{i}, \mathrm{k})_{O} \exp \left(\frac{\mathrm{j} 2 \pi \mathrm{mi}}{\mathrm{M}}\right) \exp \left(\frac{\mathrm{j} 2 \pi \mathrm{nk}}{\mathrm{N}}\right)
$$


for $\mathrm{i}=0,1, \ldots, \mathrm{M}-1 ; \mathrm{k}=0,1, \ldots, \mathrm{N}-1$

$$
\beta_{\mathrm{O}} \cdot \beta_{\mathrm{F}}=\frac{1}{\mathrm{M} \cdot \mathrm{N}}
$$

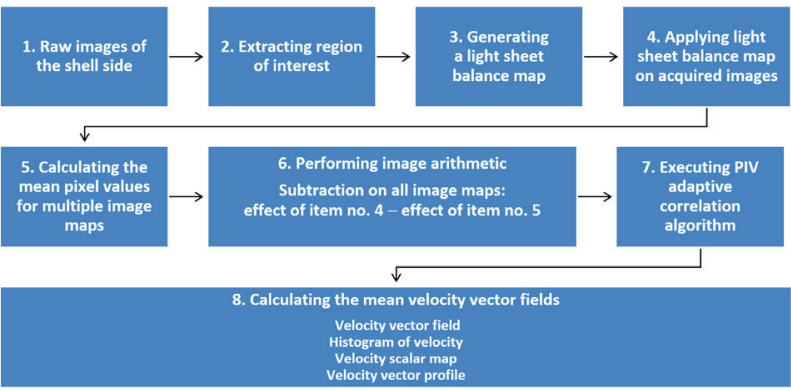

Fig. 3. Overview of the PIV methodology.

Besides the knowledge of the intervals separating the registration of both images, the determination of the vector velocity profile by application of the PIV method involved the need to get to know an arbitrary characteristic dimension of the registered image with the aim of calibrating the linear scale of the phenomenon. A familiar dimension representing the internal diameter of the internal shell of the heat exchanger was applied for this purpose.

\section{Results and discussion}

The imaging of shell side across the tube bundle in a real heat exchanger performed by the application of the proposed optical track was associated with the occurrence of the phenomenon of perspective in the tube bundle in the section from the plane of the laser sheet to the tube sheet of the heat exchanger. The effect of the phenomenon of perspective is presented in Fig. 4. The same effect was evident in the work [16].

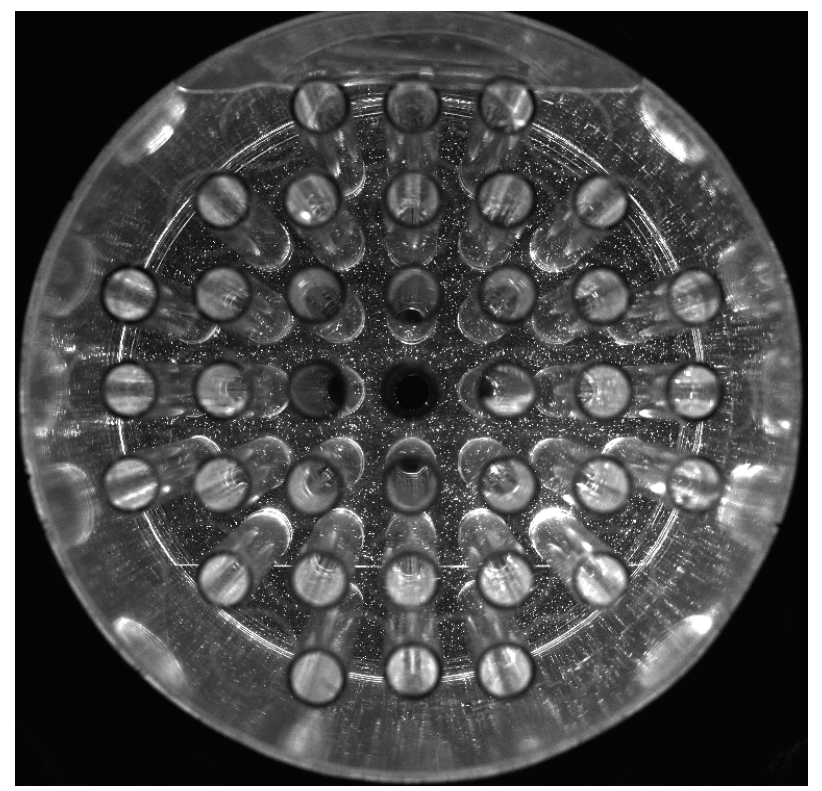

Fig. 4. Effect of the perspective in the shell-and-tube heat exchanger during across tube bundle imaging.
Perspective forms a considerable impediment in the assessment of the hydrodynamics of particular streams and imposes the limitation of the regions of interest. The perspective coefficient calculated as the ratio of the actual external tube diameter in the laser sheet plane to the diameter of the pipe end in the tube sheet captured by the image was determined to be equal to 0.84 . This corresponds to the loss of $16 \%$ of the region between the tube rows in the focal point of and the adjacent tube rows and $100 \%$ for the successive rows. The visualization of velocity vector field under such conditions is shown in Fig. 5. No visible continuity of seeding particles movement is an significant barrier in the description of flow hydrodynamics using PIV method.

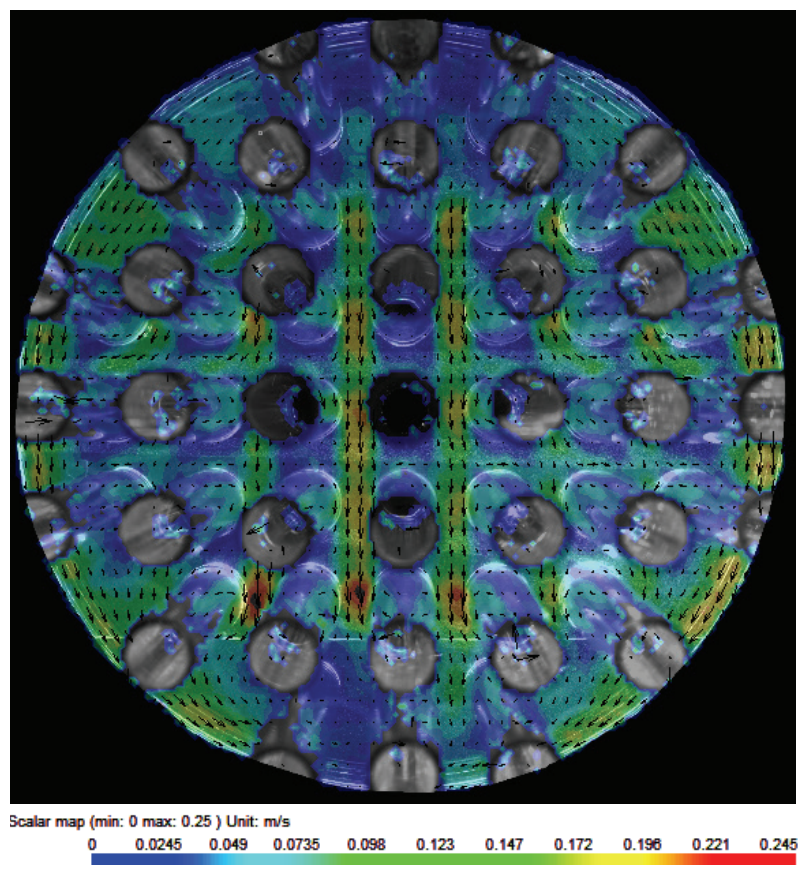

Fig. 5. Visualization of velocity vector field throughout the whole cross-section of the shell side.

As a consequence, the measurements are limited to the analysis in the tube rows that are situated in the focal point. A similar solution was used at work [16]. In the consideration of the fact that the applied measurement methodology involves the analysis of the velocity profiles in the symmetry axes of the zones located between the tube bundles and velocity vector field determined in undistorted region of interest, the loss of the zone applied to measurements did not affect the results of the analysis since calculation algorithms are not limited by the phenomenon of perspective.

The assessment of irregularity of fluid distribution during its flow across a tube bundle began by determination of the velocity distribution symmetry. This goal was achieved by determination of the velocity profile across the tube bundle in the plane across all tube rows above the $4^{\text {th }}$ row counting from the top (Fig. 6).

Velocity profiles analysis allowed to identify common areas of irregularity liquid distribution in shell side. This irregularity was manifested by the variation of 
a)

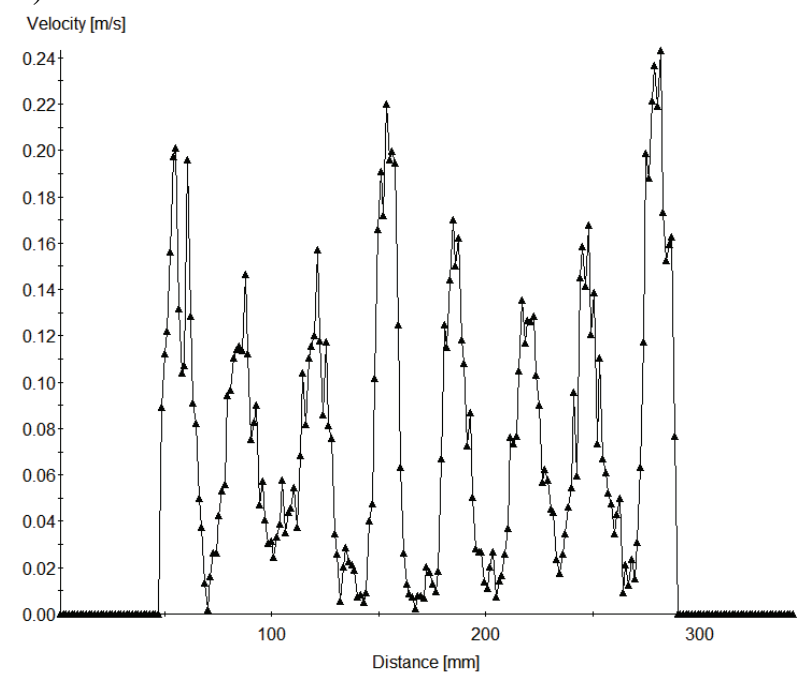

b)

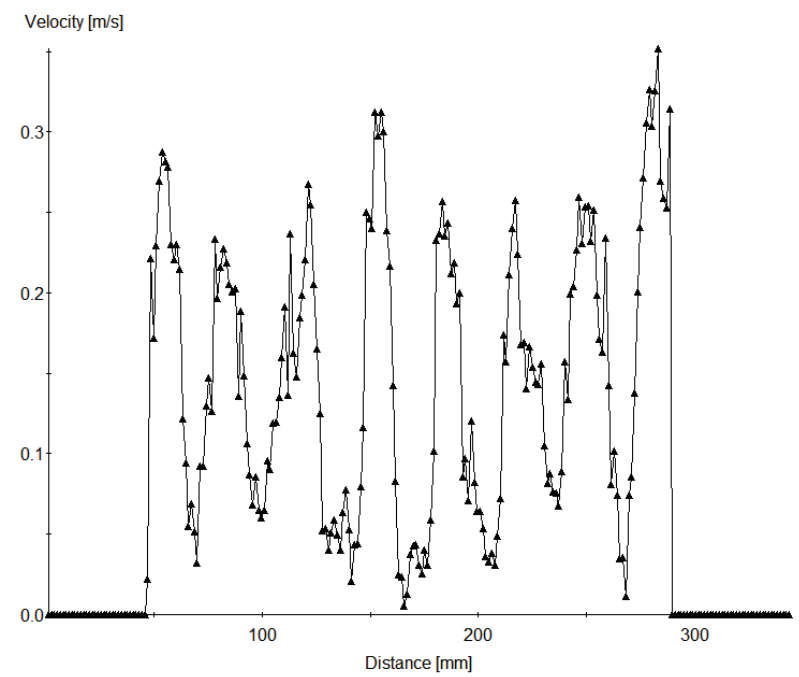

c)

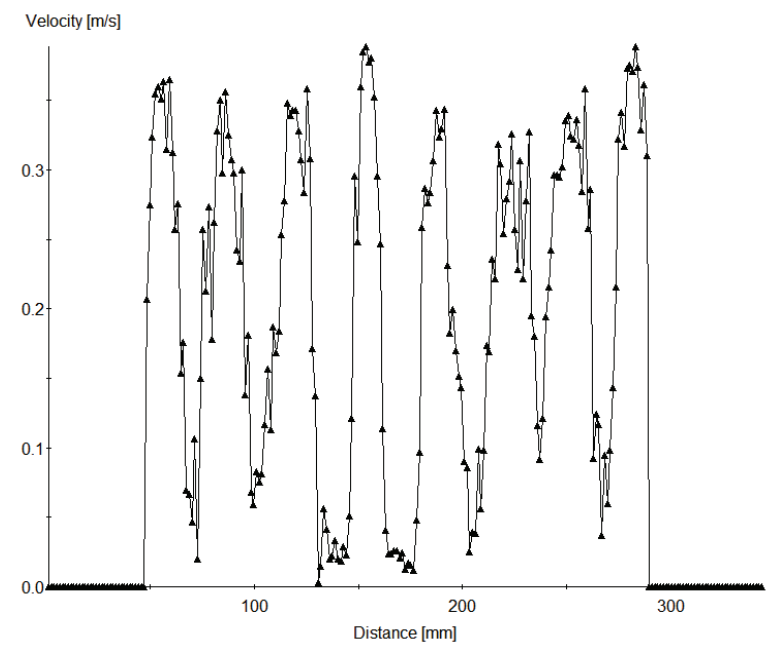

Fig. 6. Velocity profiles above $4^{\text {th }}$ tube row: a) $5 \mathrm{~m}^{3} / \mathrm{h}$, b) 7.5 $\left.\mathrm{m}^{3} / \mathrm{h}, \mathrm{c}\right) 10 \mathrm{~m}^{3} / \mathrm{h}$.

liquid flow velocity in the analogous region of the tube bundle along the horizontal axis above $4^{\text {th }}$ tubes row. Increased maximum local velocity accrue to external left and right regions of shell side from SL to 1 and from 7 to SR according tube arrangement shown in the Fig. 2. Another one high velocity region was located in the center of shell side (3-4 and 4-5 regions). In the above regions of flow velocity variation, maximum value of the local velocity, depending on flow rate, was higher of $32 \%, 22 \%$ and $8 \%$, respectively. However, the symmetrical nature of velocity variations disappears with increasing flow rate (Fig. 7). For a flow rate equal to 10 $\mathrm{m}^{3} / \mathrm{h}$, the difference in velocity variation in particular row of tubes does not exceed $10 \%$. Regions characterized by increased local velocity are also symmetrical and uniformly situated on both sides of the shell side cross-section. The symmetry axis divides the heat exchanger into left and right-hand sizes, which are similar in terms of the hydrodynamic parameters. The testing measurements and calculations can by performed in limited area to a half of the cross-section of a heat exchanger. In certain conditions, this operation can be simplified in this manner without the impact on the quality of the results. In connection with this, the further part of the study focused on the assessment of the irregularity of fluid distribution only from the 4th to the 7 th tube rows (i.e. on the left side of the transverse section of the tube bundle in the heat exchanger).

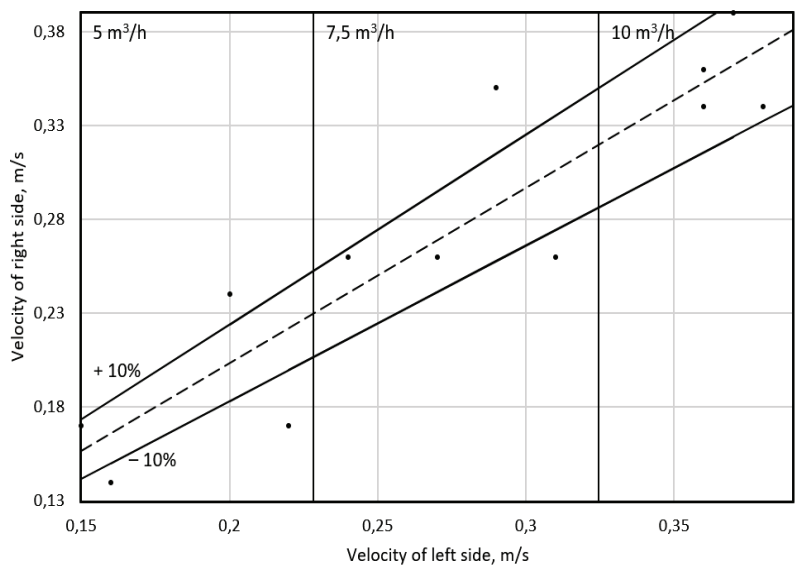

Fig. 7. Influence of flow intensity on the symmetry of liquid flow in the shell side.

The vector velocity fields for the above four cases of location in shell side are given in Table 1 and 2. On the basis of this analysis, it was possible to confirm irregularity of flow distribution by determine the trend representing the mean value of flow velocity. This procedure was performed using the histogram function (Fig. 8-10). The space between the $7^{\text {th }}$ tube row and shell is characterized by the higher value of mean flow velocity regardless of the flow rate through the heat exchanger. In this region mean value of velocity equal to: $0.19 \mathrm{~m} / \mathrm{s}, 0.29 \mathrm{~m} / \mathrm{s}$ and $0.37 \mathrm{~m} / \mathrm{s}$ in depending on increasing flow rate used in the experiment. This is due to the fact that liquid flowing around the shell of the heat exchanger does not encounter obstacles in the form of tube rows and consequently, there is no increased local pressure drop in this region and flow assumes considerable value of the mean velocity. 
Table 1. Velocity vector field in particular region of interest between rows of tubes.

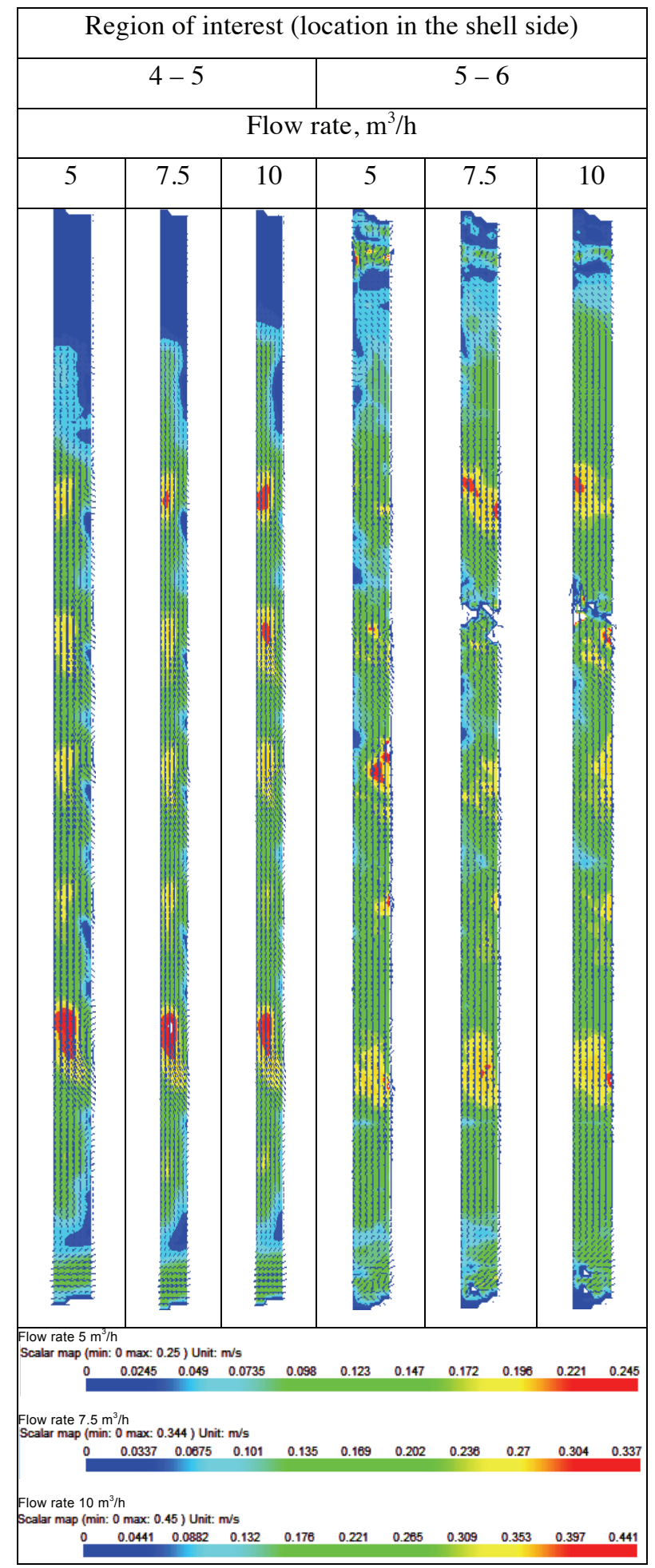

High mean value of flow velocity was also observed in the central part of the shell side (region 4-5). The mean values of flow velocity for similar flow rates were $37 \%$, $41 \%$ and $37 \%$ lower than the external region. In this case mean value of flow velocity is strongly determined by perpendicular flow to the tube bundle. The areas between these regions can be considered as a transient flow with a characteristic decrease of mean velocity value. On one hand, we have to do with an increased
Table 2. Velocity vector field in particular region of interest between rows of tubes or rows of tubes and shell.

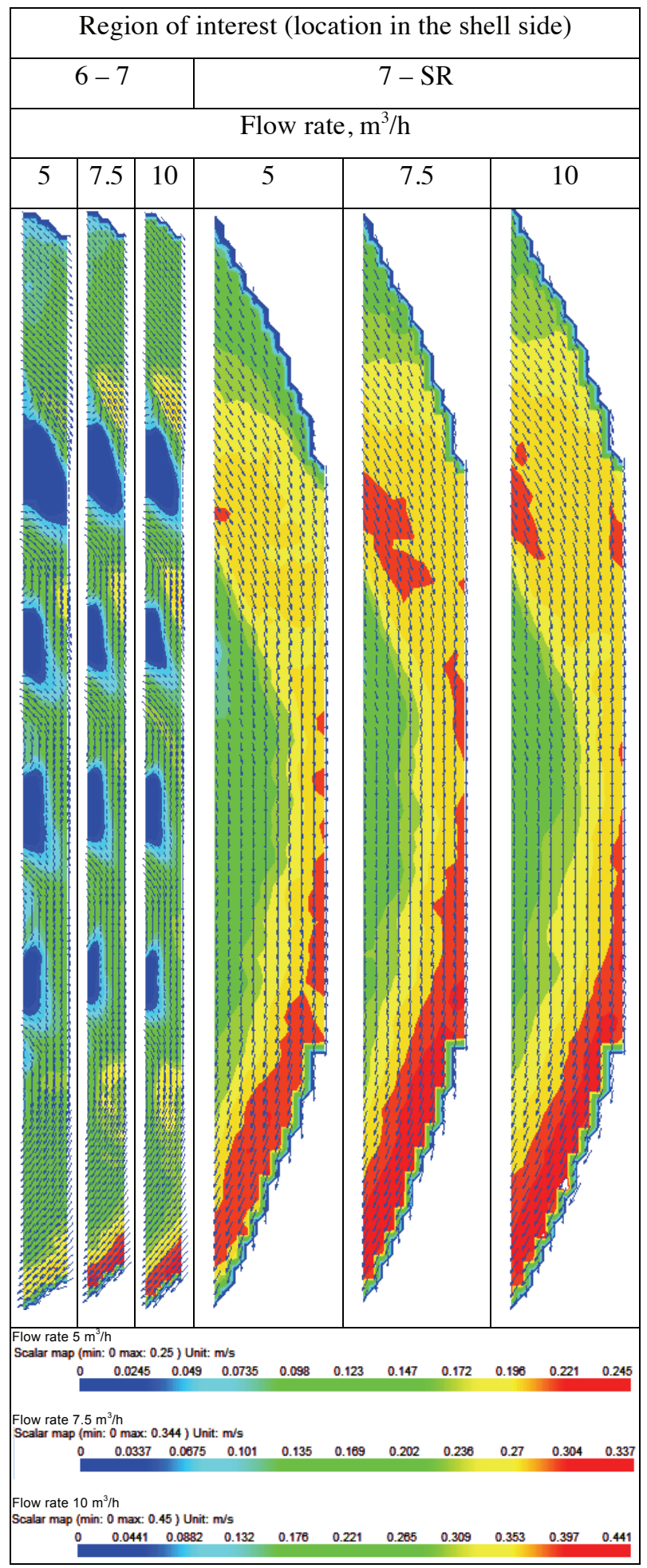

local pressure drop due to the existence of adjacent tube bundles, on the other, the liquid in this region has a lower initial velocity since the flow direction of this stream is not predominantly directed perpendicular to the tube. This demonstrates the existence of regions with various hydrodynamic characteristics. As a result, the study in this paper identified the existence of three irregularity regions. Two external regions between the last rows of tubes and the shell, the central region and 
the transition region located between the two. Irregularity distribution of liquid flow in this region can negatively affect the performance of the heat exchanger, especially at low flow rate. This applies both to the effect on the heat transfer coefficient and to the susceptibility to settling contamination in the tube bundle.

a)

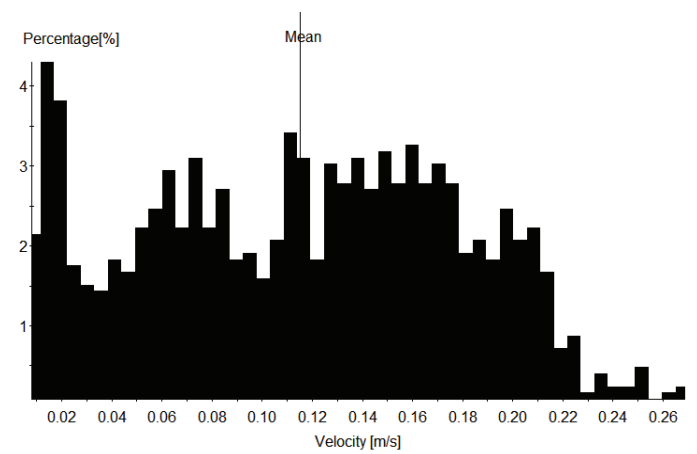

b)

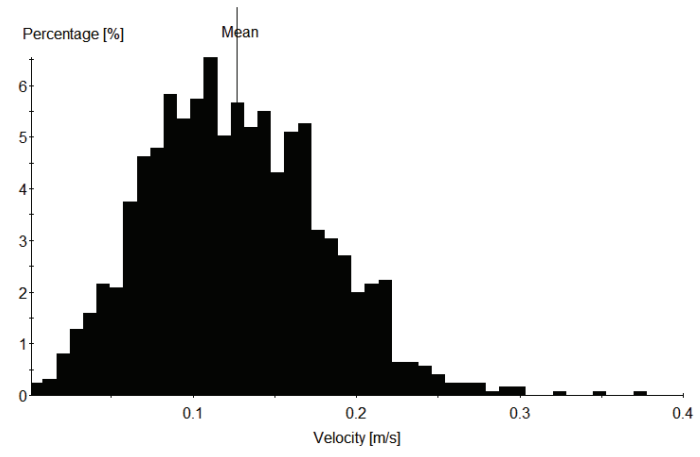

c)

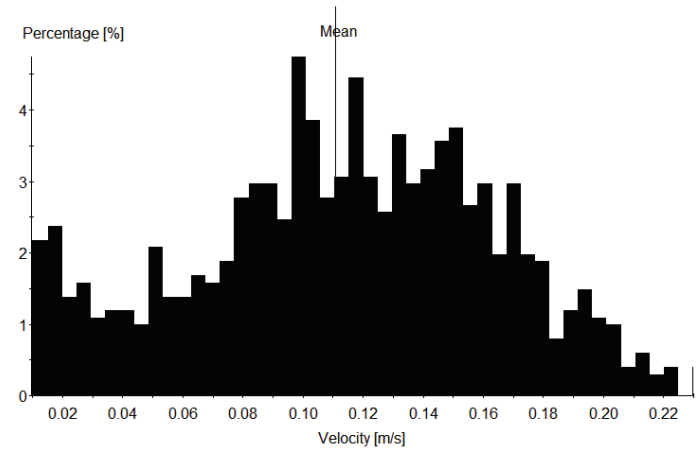

d)

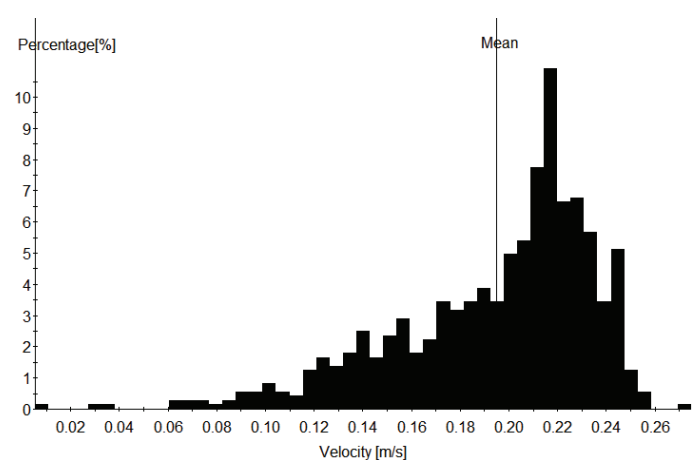

Fig. 8. Histogram of velocity for particular region of interest (flow rate $5 \mathrm{~m}^{3} / \mathrm{h}$ ): a) 4-5, b) 5-6, c) 6-7, d) 7-SR. a)

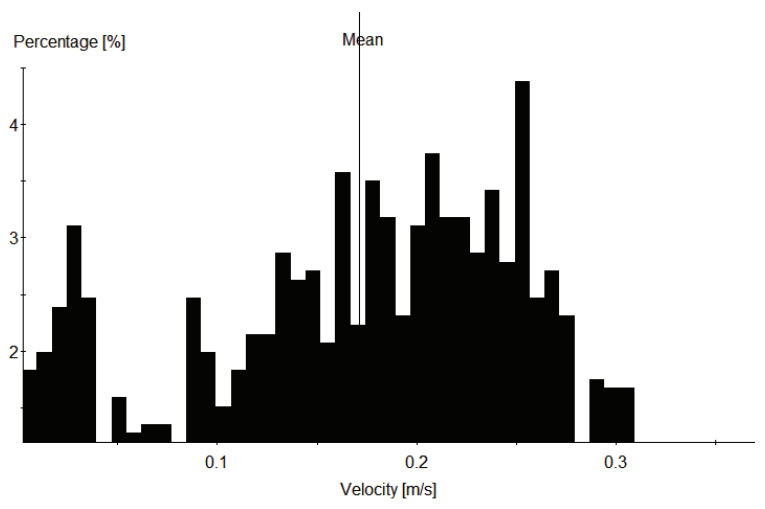

b)

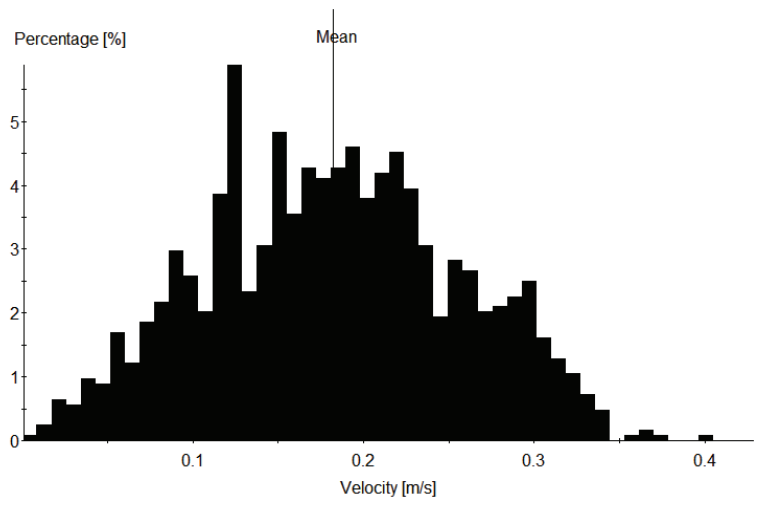

c)

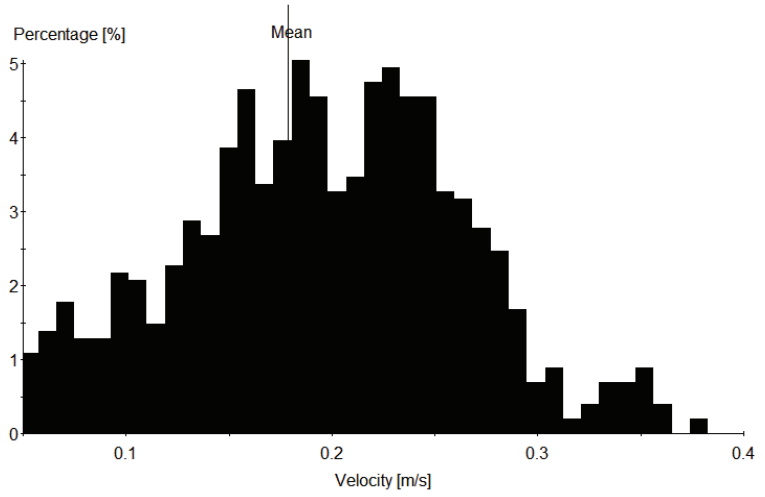

d)

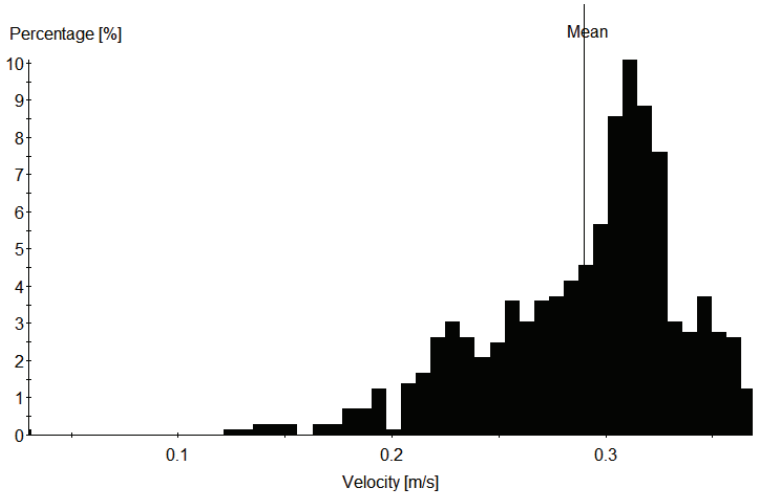

Fig. 9. Histogram of velocity for particular region of interest (flow rate $7.5 \mathrm{~m}^{3} / \mathrm{h}$ ): a) 4-5, b) 5-6, c) 6-7, d) 7-SR. 
a)

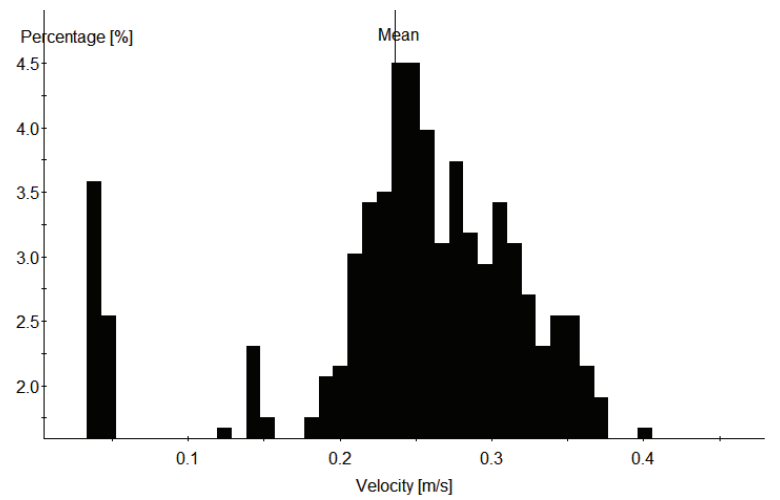

b)

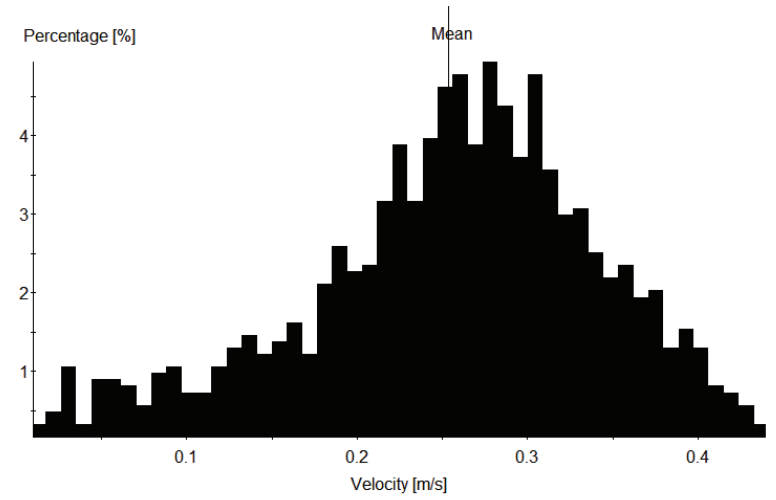

c)

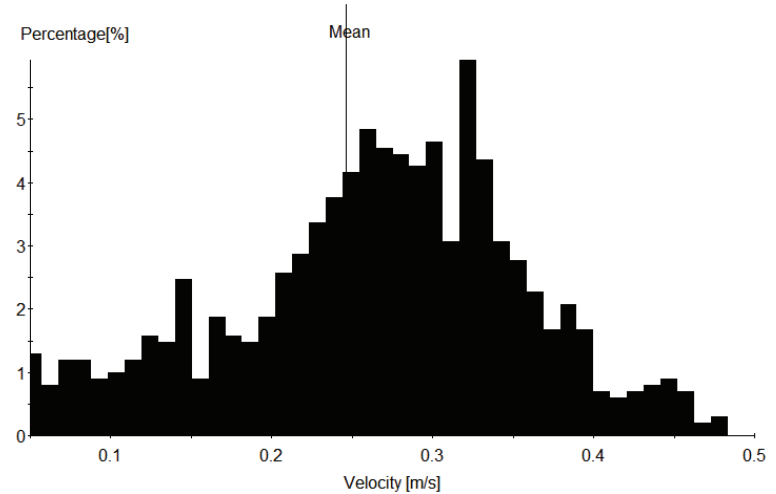

d)

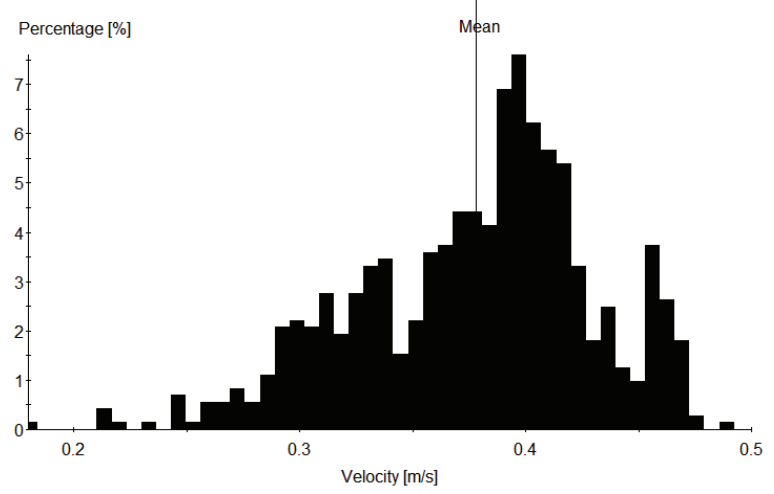

Fig. 10. Histogram of velocity for particular region of interest (flow rate $10 \mathrm{~m}^{3} / \mathrm{h}$ ):

a) $4-5$, b) 5-6, c) $6-7$, d) 7-SR.

\section{Conclusions}

The study of the hydrodynamics of the liquid flow through a tube bundle of a heat exchanger with segmental baffles confirmed the effectiveness of the application of Digital Particle Image Velocimetry (PIV) technique applied for the diagnostics of this type of apparatus. However imaging of the shell side across the tube bundle performed by proposed optical track was associated with the occurrence of the phenomenon of perspective. This phenomenon was a substantial barrier in full imaging of the liquid flow in the shell side. In order to carry out a complete assessment of the velocity vector fields across tube bundle, the investigation should be conducted on a simplified model of heat exchanger with a tube bundle ending directly behind the laser sheet. The results of the study confirmed the existence of a symmetrical flow across the entire cross-section of the tube bundle. The symmetry axis divides the heat exchanger into left and right-hand sizes, which are similar in terms of velocity vector fields parameters. The testing performed into flow that is transverse to the tube bundle orientation can be therefore limited to a half of the cross-section of a heat exchanger. In certain conditions, the procedures involving data processing and analysis can be simplified in this manner without the impact on the quality of the results. The analysis of the vector velocity fields indicated that the distribution of the mean velocities of the liquid flow varies in the particular tube rows. This demonstrates the existence of irregularity in hydrodynamic characteristics. As a result, the investigation in this paper identified the existence of three main regions of the cross-section of shell side. Two symmetrical external regions between the last rows of tubes and the shell, the central region and the transition region located between the two. Differences in local flow velocities in these regions affect the performance of the heat exchanger. The problems discussed in this paper should be confirmed throughout a more extensive study program accounting for other geometries of tube bundle and various flow rates and the relationships above with the heat transfer coefficient.

\section{References}

1. Standards of the Tubular Exchanger Manufacturer Association, (TEMA, 9th Edition, New York, 2007)

2. S. Kakacs, L. Hongtan, A. Pramuanjaroenkij, Heat Exchangers, Selection, Rating, and Thermal Design, (CRC Press, 2012)

3. E.A.D. Saunders, Features relating to thermal design in Heat Exchanger Design Handbook, (Hemisphere, Washington, 1983)

4. A.F. Jozaei, A. Baheri, M.K., Hafshejani, A. Arad, WASJ 18, 7 (2012)

5. B. Parikshit, K.R. Spandana, V. Krishna, T.R. Seetharam, K.N. Seetharamu, IJHMT 84 (2015)

6. J. Guziałowska, G. Ligus, R. Ulbrich, Proceedings of 5th International Conference on Transport 
phenomena in Multiphase Systems (Bialystok, 2008)

7. T.H. Chang, Ch. Lee, H. Lee, K.S. Lee, J. Therm. Sci. 24, 4 (2015)

8. R.J. Adrian, J. Westerweel, Particle Image Velocimetry (Cambridge University Press, New York, 2011)

9. C. Iwaki C., K.H. Cheong, H. Monji, G. Matsui, Exp. Fluids 37 (2004)

10. M. Karaś, D. Zając, R. Ulbrich, Inż. Ap. Chem., 52, 5 (2013)

11. S.S. Paul, M.F. Tachie, S.J. Ormiston, IJHFF, 28, 2 (2007)

12. Ch. Bin, G. Liejin, J. Therm. Sci. 9, 3 (2000)

13. M. Yataghene, F Fayolle, J. Legrand, ATE 31 (2011)

14. G.L. Morrison, I. Budihardjo, M. Behnia, Solar Energy 78 (2005)

15. N. Liu, W. Wang, J. Han, M. Zhang, P. Angeli, Ch. Wu, J. Gong, CES 152 (2016)

16. Sunghyuk T.K. Hyoung, W.R. Bo, J.S. Hyung, ANE, 98 (2016) 